\title{
P-Null Phenotype Due to a Rare Frame-Shift Mutation and with Allo-Anti-PP1Pk Causing a Severe Hemolytic Transfusion Reaction: A Case Report with Clinical Management
}

\author{
Ashish N. Kanani $^{a}$ Snehal B. Senjaliya ${ }^{a}$ Manisha M. Rajapara ${ }^{a}$ \\ Judith Aeschlimann ${ }^{b}$ Connie M. Westhoff ${ }^{b}$ Sanmukh R. Joshi ${ }^{a}$

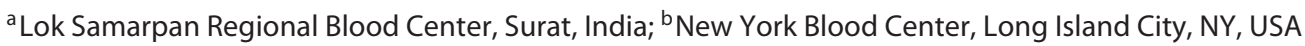

\section{Keywords}

Allo-anti-PP1Pk · Rare P-null phenotype · Hemolytic transfusion reaction $\cdot$ Clinical management

\begin{abstract}
Introduction: The identification of alloantibodies to highfrequency antigens (HFA) and subsequent transfusion management can be challenging and often poses a problem in finding the compatible blood for transfusion. The aim of this study was to investigate the specificity of the antibody to the HFA causing a hemolytic transfusion reaction (HTR) and procure the compatible blood unit for future transfusion. Case presentation: A 4-year-old female met with a head injury that led to intracranial bleeding and surgical intervention was required to remove blood clots. In the face of anemia, blood transfusion was planned. The pretransfusion tests on her blood sample revealed the presence of a pan-reactive alloantibody with hemolytic properties. She was transfused with $10 \mathrm{~mL}$ of the least incompatible red blood cells (RBCs) to which she reacted with signs of clinical hemolysis, i.e., chill, rigor, fever, and hemoglobinuria, on 3 different occasions. Despite her anemia, she was managed by medical intervention only. Her antibody reacted with all RBCs tested, except autologous and P-null ( $p$ phenotype) cells. Her RBCs did not react with anti-PP1Pk, which corroborated her phenotype as P-null. The genomic study revealed she was hemior homozygous or for a deletion of 26-bp in A4GALT exon 3, previously reported as causing the P-null phenotype and designated A4GALT*01N.019. Conclusion: This report docu-
\end{abstract}

karger@karger.com www.karger.com/tmh

Karger ${ }^{\prime \prime} \%$

BOPEN ACCESS
(C) 2021 The Author(s)

Published by S. Karger AG, Basel

This article is licensed under the Creative Commons Attribution NonCommercial-NoDerivatives 4.0 International License (CC BYNC-ND) (http://www.karger.com/Services/OpenAccessLicense). Usage and distribution for commercial purposes as well as any distribution of modified material requires written permission. ments a rare case of the P-null phenotype with an alloantiPP1Pk causing a severe HTR to transfusion of the trial dose of the least incompatible blood. The case is the first example of this specific A4GALT mutation found in India.

(C) 2021 The Author(s)

Published by S. Karger AG, Basel

\section{Introduction}

Many cases with rare blood types and/or antibodies to high-frequency antigens (HFA) of clinical significance have been reported in India [1]. Antibody identification and allocating compatible units for transfusion can be challenging, especially when blood centers and hospitals have limited resources [1]. The P-null phenotype, also referred to as the $\mathrm{p}$ phenotype (previously $\mathrm{Tj}[\mathrm{a}-]$ ), is rare, and is defined by the lack of $\mathrm{P}, \mathrm{P} 1$, and $\mathrm{Pk}$ on red blood cells (RBCs). All $\mathrm{p}$ phenotype individuals have anti-PP1Pk in the plasma, and complement-mediated hemolysis is thought to be the cause of the hemolytic transfusion reaction (HTR) [2], hemolytic disease of the fetus and the newborn (HDFN) [3], and spontaneous abortions [4] reported in these patients. PP1Pk is encoded by $A 4 G A L T$, and the P-null phenotype results from a variety of mutations at the genomic level [5]. This report describes a case that presented with an alloantibody to an HFA. The complement-mediated hemolytic properties indicated specificity towards the antigens of the H, Lewis, P1Pk, Colton, or I blood groups. An extended serological investigation identified the PP1Pk specificity, and molecular testing revealed the underlying variant allele. 


\section{Case Report}

A 4-year-old female had a fall from the first floor of her residence and met with a head injury followed by loss of consciousness. She was intubated with supportive management immediately at the nearby medical facility and later transferred to a multi-specialty hospital. On examination, her clinical condition was serious with a Glasgow Coma Scale (GCS) $<7$, her pupils were bilaterally slowly reactive, heart rate $140 / \mathrm{min}, \mathrm{SPO}_{2} 96 \%$ on ET, BP 94/40 $\mathrm{mm} \mathrm{Hg}$, and there was a hematoma over the inferior parietotemporal area. On admission, she was subjected to CT brain scan. The required treatment for raised intracranial pressure (ICP) was started and decompressive craniotomy was performed within $6 \mathrm{~h}$ by the neurosurgeon. Postoperative treatment for raised ICP continued with minimal ventilator support; ionotropes for hypovolemic shock, antibiotic, and anticonvulsants were given.

In the face of moderate anemia, i.e., a hemoglobin $(\mathrm{Hb})$ value of $6.2 \mathrm{~g} / \mathrm{dL}$, blood transfusion was indicated and a blood sample collected in EDTA anticoagulant was sent to the blood center with the detail that she had no history of blood transfusion. She was group $\mathrm{A}_{1} \mathrm{RhD}$-positive on forward-grouping but the reversegrouping showed discrepant results. The discrepancy was attributed to the presence of an alloantibody detected on the antibody screening test (AST). The antibody was pan-reactive as it reacted with all 11 cells on the antibody identification panel. Besides this, it reacted with 86 random group A and O RBCs as well as 1 example of Bombay phenotype. The antibody reacted in saline lowionic-strength solution (LISS) tube IAT, gel-card (Bio-Rad/DiaMed GmbH, Cressier, Switzerland), glass-beads column agglutination (Ortho Clinical Diagnostics, Pencoed, UK), and solid-phase (Neo, Immuncor, Norcross, GA, USA) assays. These observations explained the anomaly in the reverse grouping. Reaction grade varied from +1 to +3 , with partial hemolysis in the saline phase and +3 to +4 agglutination in LISS-IAT phase. The titer by saline tube was 1:4. Both the parents and her sibling were incompatible.

The antibody specificity to Lewis and I antigens was ruled out by hemagglutination inhibition test, using soluble antigens from plasma and milk, respectively. The antibody was partially inhibited by hydatid cyst fluid, suggesting specificity to the antigens within the P1Pk blood group. The antibody reacted equally well with the adult and cord RBCs, further ruling out anti-I and antii. In view of the complement-mediated hemolytic nature of the antibody, we suspected anti-Co3 or anti-PP1Pk specificity. AntiCo3 specificity was ruled out as the antibody reacted with 1 example of RBCs with the Colton null phenotype. Anti-PP1Pk was confirmed when her plasma was found to be compatible with Pnull ( $\mathrm{p}$ phenotype) RBCs and her RBCs were nonreactive with an example of anti-PP1Pk plasma (Table 1).

Meanwhile, her $\mathrm{Hb}$ dropped to $3.8 \mathrm{~g} / \mathrm{dL}$, so her physician decided to transfuse the least incompatible blood units made available from another blood bank. The patient showed signs of clinical hemolysis with chill, rigor, fever, and hemoglobinuria upon transfusion of approximately $10 \mathrm{~mL}$ of the least incompatible RBCs on 3 different occasions over a span of 2 consecutive days.

The genomic study was carried out by first looking for the mutation found in an earlier case [6] that came from the same geographic vicinity as our patient, considering a possible founder effect for the same mutation. As these results were negative, further testing was performed. DNA was isolated from her white blood cells (WBCs). The A4GALT coding sequence (exon 3) was amplified and the presence of an amplicon of the expected size was confirmed by agarose gel electrophoresis. The purified products were Sanger-sequenced and aligned to the reference se-
Table 1. Identification of the patient's P-null phenotype by the 2 column agglutination devices

\begin{tabular}{lccccc}
\hline \multirow{2}{*}{ Test RBCs } & \multicolumn{2}{l}{ Patient's plasma } & & \multicolumn{2}{l}{ Anti-PP1Pk } \\
\cline { 2 - 3 } \cline { 5 - 6 } \cline { 5 - 6 } & Ortho & Bio-Rad & & Ortho & Bio-Rad \\
\hline 1. PP1Pk-negative donor & 0 & 0 & & 0 & 0 \\
2. Patient (autologous) & 0 & 0 & & 0 & 0 \\
3. PP1Pk-positive donor & +4 & +3 & & +3 & $+2-3$ \\
\hline
\end{tabular}

quence NG_007495.2 with ClustalX. Sequencing of the A4GALT exon 3 found that the sample appeared to be homozygous for c.972_997del deletion, previously reported as A4GALT*01N.19 [7]. However, we are not able to rule out the rare possibility that the allele in trans carried a large inactivating deletion and that the sample was hemizygous. This $26-b p$ deletion results in a frameshift and extends the open reading frame for 83 amino acids followed by a stop codon (p.Arg325Alafs*113) (Fig. 1).

\section{Discussion}

The P blood group system is polymorphic in nature, comprising $\mathrm{P} 1$ as a strongly expressed antigen, $\mathrm{P} 2$ lacking the $\mathrm{P} 1, \mathrm{P}$ as the high frequency antigen, and $\mathrm{Pk}$ as the precursor for all [8-10]. The absence of these antigens comprises the $\mathrm{P}$-null phenotype, with a presence of regularly/ naturally occurring alloantibody directed to the high-frequency antigen complex of the PP1Pk [2]. The P-null phenotype is a rare entity that occurs in different parts of the world [11-14], which makes it difficult to find compatible blood for transfusion. Our case posed a tremendous problem, i.e., to find compatible blood in an emergency situation. In India, a few suspected cases of P-null phenotype have been recorded, but only 1 case was confirmed by molecular analysis [6]. The case presented here is, therefore, the second example of P-null to be reported in India.

Anti-PP1Pk is known to show complement-mediated hemolysis in vitro and clinical significance to cause HTR, HDFN, or spontaneous abortion [2-4]. The antibody in our patient also showed in vitro lysis of incompatible RBCs and yielded severe HTR to a small volume of incompatible RBCs transfused, presenting a picture similar to the previously described case [2]. Anti-PP1Pk in our case was partially inhibited by hydatid cyst fluid, subscribing to the $\mathrm{Pk}$ and $\mathrm{P} 1$ antigens on RBCs having the same terminal disaccharide $(\mathrm{Gal}[\alpha 1 \rightarrow 4] \mathrm{Gal})$ as a soluble entity in the hydatid cyst fluid [15].

While A4GALT encodes the transferases that catalyze the synthesis of $\mathrm{P} 1$ and $\mathrm{Pk}$ [16], the $\mathrm{P}$ antigen is encoded by a different gene called B3GALNT1 [17]. However, antigen expression is also affected by genetic mutations in A4GALT because $\mathrm{Pk}$ is the precursor of $\mathrm{P}$ [10]. Even though rare, there are $>35$ reported $A 4 G A L T$ variants that 


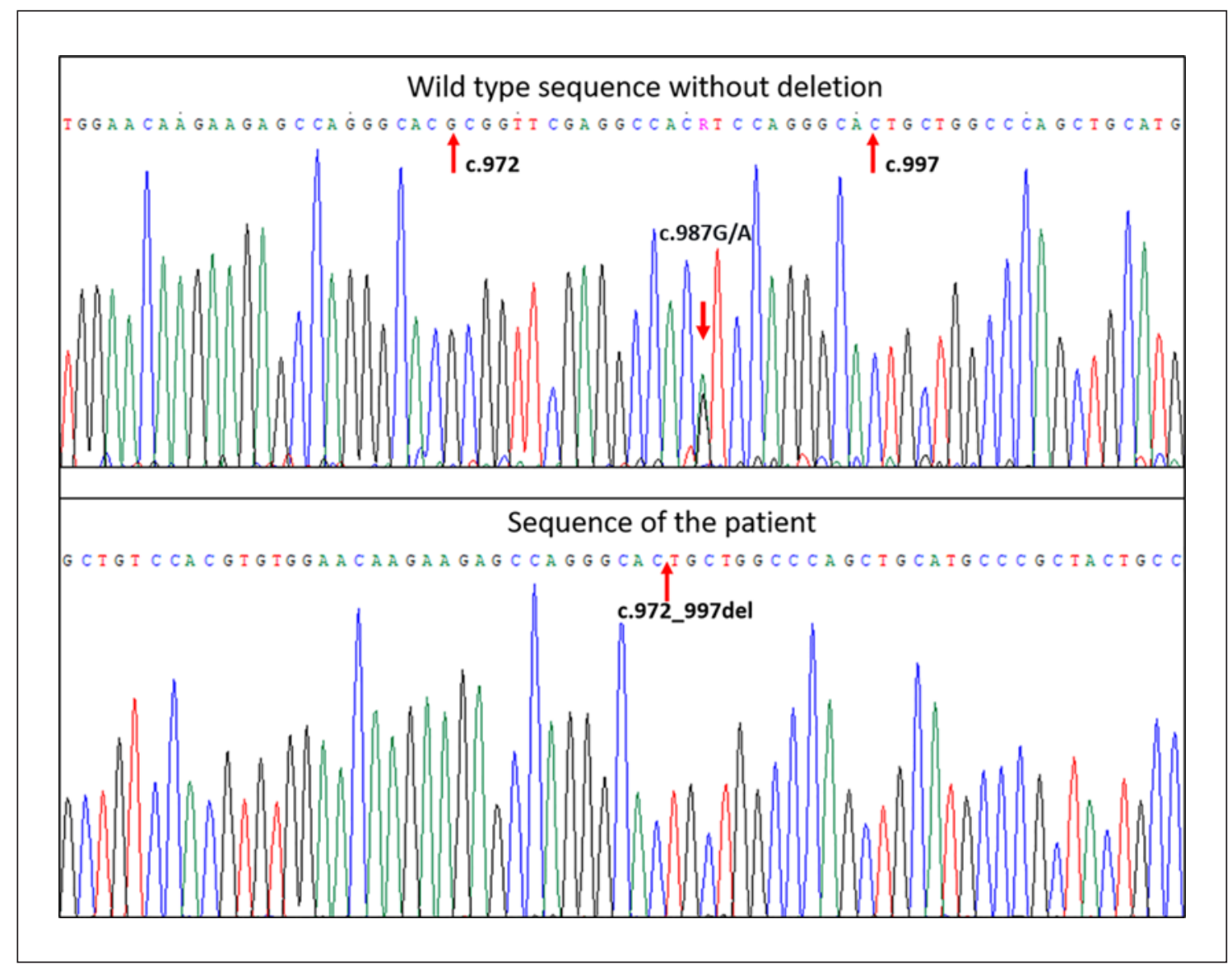

Fig. 1. Electropherogram of the consensus sequence (top) and proband sequence (bottom), indicating area of deletion (red arrows) showing the location of the 26-bp deletion c. 972_997del. The consensus sequence is heterozygous for a common silent change (c.987G>A).

cause the P-null phenotype [18]. The genomic backgrounds of these null alleles are heterogeneous; missense mutations in conserved regions of the galactosyltransferase gene are predicted to lead to an inactivation of the enzyme $[9,10]$, and nonsense mutations like nucleotide insertions or deletions lead to a change in the reading frame and, most likely, to a total loss of enzyme activity [9]. Xu et al. [7] reported a case with a 26-bp deletion at position c.972_997 of A4GALT causing a shift of the reading frame, designated as $A 4 G A L T^{*} 01 N .19$. In our patient, we found the same variant allele, the first of its kind in an Indian person, i.e., of distinctly different race/ethnicity from the previous report, which was of a Chinese patient.

\section{Conclusion}

This report documents a rare case of the P-null phenotype with an alloanti-PP1Pk causing severe HTR to transfusion of a trial dose of the least incompatible blood. The case is the first example of this specific A4GALT mutation encountered in India.

\section{Acknowledgement}

The authors thank Ms. Christine Lomas, New York Blood Center, Long Island, for sharing RBCs with the P-null phenotype and anti-PP1Pk from her collections to confirm the phenotype and antibody specificity of our case. We also thank Dr. Shamee Shastry and Dr. Manjunath Joshi, Manipal Academy of Higher Education, Manipal, for organizing and testing our patient's sample to rule out the mutation involved in the previously reported case from India.

\section{Statement of Ethics}

The study was approved by the committee members of the Institute to carry out this research. The patient being a minor, written informed consent was obtained from her father for publication of this case report. The patient's identity is not disclosed in the paper.

\section{Conflict of Interest Statement}

The authors have no conflicts of interest to disclose. 


\section{Funding Sources}

The authors did not receive any funding.

\section{Author Contributions}

A.N.K., S.B.S., M.M.R., and S.R.J. were involved in the serological diagnosis of the rare phenotype and the patient blood management; J.A. and C.M.W. performed the molecular work and interpretation of results; S.R.J., J.A., and C.M.W. drafted and critically evaluated the manuscript.

\section{References}

1 Reesink HW, Engelfriet CP, Schennach H, Gassner C, Wendel S, Fontão-Wendel R. et al. Donors with a rare pheno (geno) type. Vox Sang. 2008 Oct;95(3):236-53.

2 Levine P, Bobbitt OB, Waller RK, Kuhmichel A. Isoimmunization by a new blood factor in tumor cells. Proc Soc Exp Biol Med. 1951 Jul; 77(3):403-5.

3 Levine C, Sila R, Rudolphson V. Hemolytic disease of the newborn due to anti-PP1Pk (anti-Tja). Transfusion. 1977;17(6):569-78.

4 Cantin G, Lyonnais J. Anti-PP1Pk and early abortion. Transfusion. 1983 Jul-Aug;23(4): 350-1.

5 Ha C, Choi S, Yu H, Chun S, Kim KH, Lee JH, et al. First Case in Korea of a Patient with Anti-PP1Pk Antibodies: Successful Blood Management via Acute Normovolemic Hemodilution. Ann Lab Med. 2019 Nov;39(6):602-5.

6 Shastry S, Satyamoorthy K, Acharya KV, Reddy VR, Mohan G, Deepika C, et al. Deletion in the A4GALT Gene Associated with Rare "P null" Phenotype: The First Report from India. Transfus Med Hemother. 2020 Apr;47(2): 186-9.
7 Xu X, Hong X, Ma K, Lan X, Chen S, Liu Y, et al. A rare $p$ phenotype caused by a 26 -bp deletion in a 1,4-galactosyltransferase gene. Zhonghua Yi Xue Yi Chuan Xue Za Zhi. 2013 Jun;30(3):309-12. Chinese.

8 Landsteiner K, Levine P. Further observations on individual differences of human blood. Proc Soc Exp Biol Med. 1927;24(9):941-2.

9 Matson GA, Swanson J, Noades J, Sanger R, Race RR. A new antigen and antibody belonging to the P blood group system. Am J Hum Genet. 1959 Mar;11(1):26-34.

10 Fellous M, Gerbal A, Tessier C, Frezal J, Dausset J, Salmon C. Studies on the biosynthetic pathway of human P erythrocyte antigens using somatic cells in culture. Vox Sang. 1974;26(6):518-36.

11 Race RR, Sanger R. Blood Groups in Man. 6th ed. Oxford, UK: Blackwell Scientific Publications; 1975. p. 154.

12 Cedergren B. Population studies in northern Sweden. IV. Frequency of the blood type p. Hereditas. 1973;73(1):27-30.

13 Miwa S, Matuhasi T, Yasuda J. p phenotype in two successive generations of a Japanese family. Vox Sang. 1974;26(6):565-7.
14 Levene C, Shinar E, Yahalom V. Rare blood group p Tj(a-) in Israel 1975-1999 (abstract). Vox Sang. 2000;78S1:P010.

15 Klein HG and Anstee DJ. Editors. Mollison's Blood Transfusion in Clinical Medicine 12th ed, Wiley Blackwell; 2014, Oxford, UK, p150.

16 Thuresson B, Westman JS, Olsson ML. Identification of a novel A4GALT exon reveals the genetic basis of the $\mathrm{P} 1 / \mathrm{P} 2$ histo-blood groups. Blood. 2011 Jan;117(2):678-87.

17 Okajima T, Nakamura Y, Uchikawa M, Haslam DB, Numata SI, Furukawa K, et al. Expression cloning of human globoside synthase cDNAs. Identification of beta $3 \mathrm{Gal}-\mathrm{T} 3$ as UDP-N-acetylgalactosamine:globotriaosyl ceramide beta 1,3-N-acetylgalactosaminyltransferase. J Biol Chem. 2000 Dec;275(51): 40498-503.

18 [Internet] International Society of Blood Transfusion, Amsterdam, The Netherlands. working-parties/red-cell-immunogeneticsand-blood-group-terminology [cited $24 \mathrm{Aug}$ 2020]. Available from: https://www.isbtweb. org/ 